\title{
INITIAL DEVELOPMENT OF OC-BASED FLIGHT DYNAMICS ENGINEERING SIMULATOR
}

\author{
SHUHAIMI MANSOR \& AMIR FADHIL YAACOB \\ Aeronautics Group, Faculty of Mechnical Engineering \\ Universiti Teknologi Malaysia \\ Skudai, Johor.
}

\begin{abstract}
Flight simulation had played a very important role in aerospace and aviation industries as an engineering tool and training equipment. Computers provided the capability of simulating an aircraft in flight without actually flying the aircraft, thus providing safety to pilots and also cost reduction in pilot training and aircraft design. Aerodynamic characteristics were modelled mathematically to display the air craft orientation and the aircraft motion during manoeuvres. Implementation of aerodynamic database system enables a flight simulator to simulate many types of aircraft manoeuvres.
\end{abstract}

\section{INTRODUCTION}

The objective of a flight simulator is to reproduce on the ground the behaviour of an aircraft in flight. When used for research, flight simulators allow designers to explore the implications of different design options without having to incur the expense and delay arising from building and testing a range of prototypes. Flight simulation has provided the means of evaluating the likely behaviour and consequences arising from abnormal operating configurations. The Head Up Display (HUD) is a very useful device initially developed for fighter aircraft. It provides the pilot with flight data such as heading, flight attitude, airspeed and altitude of the aircraft without the pilot losing focus outside the cockpit [5]. It is a simple form of graphic representation and easy to use in program development and evaluate the aircraft motion and responses.

\section{THE SIMULATION PROGRAM}

The software used for programming purpose is Turbo Pascal version 5.5 from Borland International. It is a powerful, integrated applications development package that includes a compiler, an editor and a library manager. It is a high-level programming language and since it can access both PC hardware and the operating system itself, Turbo Pascal is well suited for many low-level systems programming tasks.

The simulations program was written in a modular-type from by using Turbo Pascal units. The simulation program consisted of a main controlling program and four other units for reading input, aerodynamic modelling and graphics. The aerodynamic characteristic of various type of aircraft were stored in data files in text form. An additional program was also written to convent nondimensional aerodynamic derivatives to their dimensional form that can be directly used by the simulation program.

\section{AERODYNAMIC MODELLING}

The aircraft dynamics is done through the equations of motion which consists of simple reduced order equations of motion. Only aerodynamic effects were taken into considerations, neglecting 
thrust and gravitational forces. These relevant equations are :

For the longitudinal motion:

$$
\begin{aligned}
& u=u_{0}+\left(X_{u} U_{0}+X_{w} w_{0}-g \theta_{0}\right) d t \\
& w=w_{0}+\left(Z_{u} U_{0}+Z_{w} w_{0}+U t o t q_{0}+Z_{\eta} \eta\right) d t \\
& q=q_{0}+\left(M_{w} w_{0}+M_{q} q_{0}+M_{\eta} \eta\right) d t
\end{aligned}
$$

For the lateral motion:

$$
\begin{aligned}
& p=p_{0}+\left(L_{p} p_{0}+L_{r} r_{0}+L_{\xi} \xi\right) d t \\
& r=r_{0}+\left(N_{p} p_{0}+N_{r} r_{0}+N_{\xi} \xi\right) d t
\end{aligned}
$$

Aircraft orientation is the aircraft flight attitude angles such as roll angle, pitch angle and yaw or more correctly azimuth angle that changes during aircraft manoeuvres. This program is a three-axis simulator, thus angular coupling have to be considered.

The equations for three-axis orientation are:

$$
\begin{aligned}
& \theta=\theta_{0}+\left(q_{0} \cos \psi_{0}\right) d t \\
& \phi=\phi_{0}+\left(p_{0} \cos \theta_{0}\right) d t \\
& \psi=\psi_{0}+\left(r_{0} \cos \theta_{0}-q_{0} \sin \psi_{0}\right) d t
\end{aligned}
$$

\section{CONTROLLING THE SIMULATION PROGRAM}

The simulation program uses control surfaces input from the computer keyboard by using the arrow keys in the same way as using a control stick of an aircraft. The problem with the keyboard input is that the single keystroke input is a pulse at a constant value, making controlling the simulated aircraft difficult, especially for fast responding and unstable aircraft. The control surfaces input magnitude from the keyboard can be changed by pressing the Ins key. The program is excited by pressing the Esc key.

The simulation program can also use a joystick for input provided that the codes for reading the input from the joystick are included. In the UTM fixed based flight simulator project an arrangement of a contact less joystick with signal amplifier and an Analog to Digital Converter PC card was used for input [4].

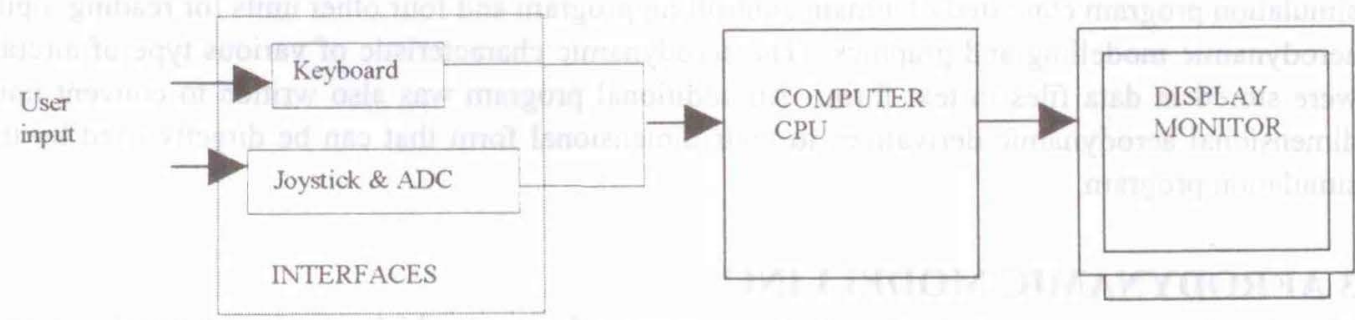

Figure 1 System Arrangement 


\section{AERODYNAMIC DATABASE FOR FLIGHT SIMULATOR}

The purpose of the aerodynamic database of aircraft is to 'fly' different types of aircraft using one simulator program. Different types of aircraft has different dynamic responses, for example a fighter aircraft has faster control input responses and low damping while transport aircraft is slow in responding but high in damping.

Text files were used to store data that can be easily understood and modified to investigate how different values of aircraft coefficient affect the dynamic responses. The aircraft have to be chosen in the beginning of the program execution. When the simulation program starts, a list of available aircraft names would be displayed. To choose an aircraft, the user just have to type the aircraft name using the listed spelling.

The aerodynamic coefficients used for this flight simulation is the dimensional type. The assumptions for flight conditions used by this simulator are:

- Thrust is assumed constant with initial speed given

- Aircraft is flying at constant altitude with a given initial altitude.

The dimensional aerodynamic coefficients can be calculated from existing aircraft non-dimensional derivatives or from a new design, obtained from stability calculations. An aerodynamic database file creation program was written to ease the conversion from non-dimensional aerodynamic derivatives to their dimensional form [1]. Thus, this simulation program is capable of simulating the dynamic flight stability characteristics of new designs by giving the input required by the aerodynamic database program.

When the simulation program is run, the new aerodynamic database file will be included in the list of aircraft and can be used directly without any modification.

$\underline{\text { Summary of longitudinal derivatives }}$

$$
\begin{array}{lll}
X_{u}=\frac{-\left(C_{D u}+2 C_{D 0}\right) Q S}{m u_{0}} & X_{w}=\frac{-\left(C_{D \alpha}-C_{L D}\right) Q S}{m u_{0}} & Z_{u}=\frac{-\left(C_{L u}+2 C_{L O}\right) Q S}{m u_{0}} \\
Z_{w}=\frac{-\left(C_{L \alpha}+C_{D 0}\right) Q S}{m u_{0}} & Z_{\eta}=-C_{Z \eta} Q S / m & M_{u}=\frac{C_{m u}(Q s c)}{u_{0} I_{y}} \\
M_{w}=\frac{C_{m \alpha}(Q s c)}{u_{0} I_{y}} & M_{q}=\frac{C_{m q}(Q s c)}{u_{0} I_{y}} & M_{\eta}=\frac{C_{m \eta}(Q s c)}{u_{0} I_{y}}
\end{array}
$$

$\underline{\text { Summary of lateral derivatives }}$

$$
\begin{array}{ll}
L_{p}=\frac{Q S b^{2} C_{l p}}{2 I_{x} u_{0}} & L_{r}=\frac{Q S b^{2} C_{l r}}{2 I_{x} u_{0}} \\
L_{\xi}=\frac{Q S b C_{l \xi}}{I_{x}} & N_{\zeta}=\frac{Q S b C_{n \zeta}}{I_{z}} \\
N_{p}=\frac{Q S b^{2} C_{n p}}{2 I_{x} u_{0}} & N_{r}=\frac{Q S b^{2} C_{n r}}{2 I_{x} u_{0}}
\end{array}
$$




\section{RESULT}

In the HUD, the symbol of the aircraft is a static object, the graphic that move and change are the pitch bars indicating the rolling and pitching angle, the heading scale indicating the compass heading of aircraft flight path, the airspeed and the barometric altitude scales with numerical values. These are several standard HUD symbology such as the FAA Symbol Set and the British BLEU symbology. The HUD graphics should contain sufficient information but not too cluttered to detract the advantage of the HUD. As for the symbology, it must convey as much information as critically possible, but without cluttering the display. For this simulation program, the British BLEU symbol set was used for the HUS [2]. Figure 1 illustrates simple HUD used primarily for the aircraft dynamics demonstration.

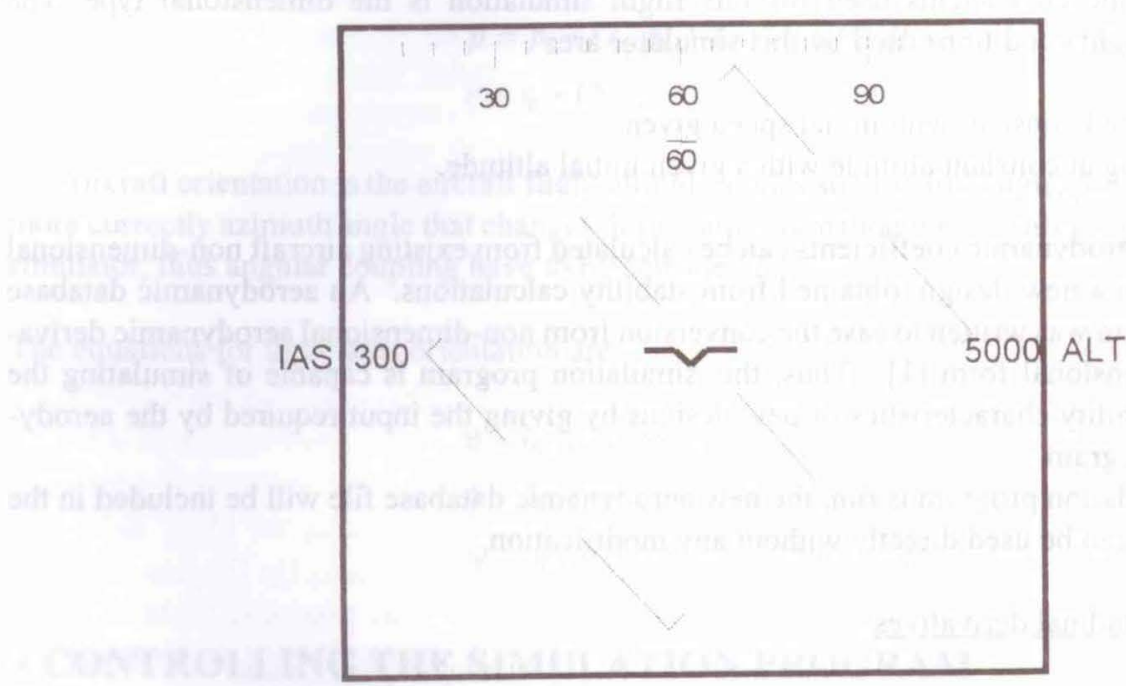

Figure 2 Dynamics Demonstration Head Up Display

The aircraft pitch and roll attitude are shown by the pitch bars, with the centre bar represents the horizon and the upper bar indicates nose pointing upward at +20 degrees of pitch angle while the lower bar indicate nose pointing downward at -20 degrees of pitch angle. Figure 3 shows the orientation between the pitch bars and aircraft during pitching motion.

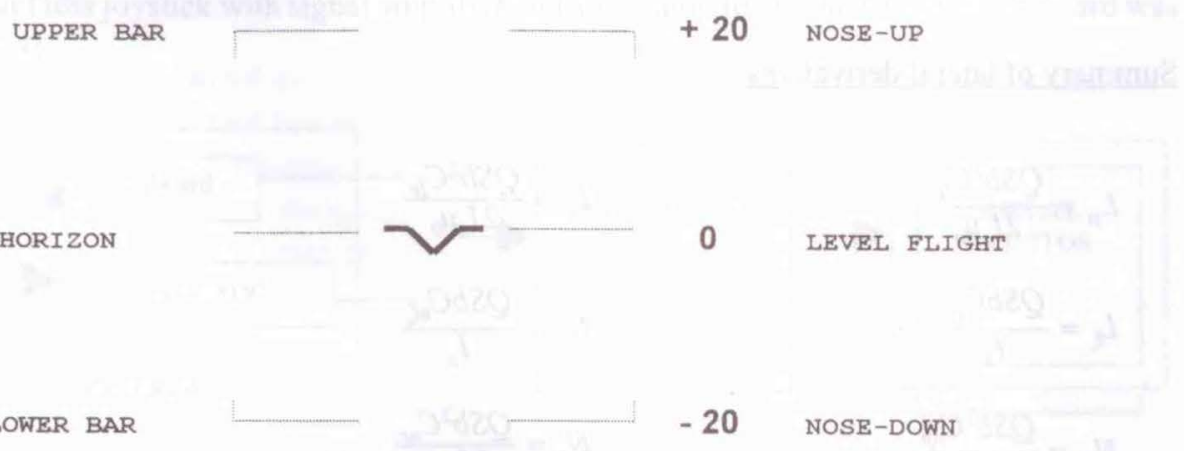

Figure 3 Orientation between pitch bars and aircraft pitching motion 


\section{CONCLUSIONS AND RECOMMENDATIONS FOR FUTURE DEVELOPMENT}

The ground-based flight simulator has been known to be the Aeronautic study and flight training easier by reducing operation and design costs, with virtually the same results and greater safety. The effectiveness of using flight simulator depends on how realistic the flight simulation can provide including the aircraft responses to control input and atmospheric conditions such as turbulence, wind shear, clear air turbulence and other factors that can contribute to the aircraft aerodynamic characteristics.

The PS-based flight simulator be able to simulate the aircraft manoeuvres with more realistic and standard form of HUD symbology. The development of aerodynamic database allow a user to choose five type of aircraft varies from highly manoeuvre fighter aircraft to slow manoeuvre big transport aircraft [3]. The modular type of software development was applied through out the project in order to make the software much easier to understand for future up-grade or modification.

For further development, there are several recommendations can be implemented. Improvement can be made to the lateral equations of motion to include the Dutch Roll Mode, Spiral Mode and side slipping movements. Inclusion of thrust effects and static stability characteristics such as stalling effects and effects of the angle of attack and addition of atmospheric affects into the simulation such as turbulence, wind shear and gust would make to simulation program more realistic. Implementation of feedback control system design can be made to improve the flying and handling qualities of aircraft stability and control through computer modeling and simulation.

\section{REFERENCES}

[1] Nelson, Robert C., 'Flight Stability and Automatic Control', McGraw-Hill 1989

[2] Rolfe, J.M. and Staples, K.J., 'Flight Simulation', Cambridge University Press, 1986.

[3] Yaacob, A. F., 'Aerodynamic Database For Flight Simulator', Aeronautics Group, Faculty of Mechanical Engineering, Universiti Teknologi Malaysia, Skudai, 1997.

[4] Perumal, K.S., 'Computer Graphic Display of Aircraft Head-Up Display', Aeronautics Group, Universiti Teknologi Malaysia, Skudai, 1996.

[5] Ringenbach, D.P., Louton, S.E, and Gleason, D., 'Use of Simulation in the USAF Test Pilot School Curriculum', Air Force Flight Test Center, Edward AFB, California 93523 USA.

\section{LIST OF SYMBOLS AND ABBREVIATIONS}
$x, y, z$
- Rolling, Pitching and Yawing axis.
$p, q, r$
- Angular rate of $x, y$ dan $z$ axis directions.
$\phi, \theta, \psi$
$\eta, \xi$
Utot
- Roll (bank), Pitch and Yaw (azimuth) angles.
- Control surface angles (elevator and aileron).
$u, v, w$
$X_{u}, X_{w}$
$Z_{u}, Z_{w}, Z_{\eta}$
$L_{p}, L_{r}, L_{\zeta}$
$M_{w}, M_{q}, M_{\eta}$
$N_{p}, N_{r}, N_{\zeta}$
$I_{x}, I_{y}, I_{z}$
- Initial flight airspeed.
- Airspeed about $x, y, z$ axis.
- Dimensional force derivatives of $x$-axis due to change in $u$ and $w$ velocities.
- Dimensional force derivatives of $\mathrm{z}$-axis due to change in $\mathrm{u}, \mathrm{w}$ and $\eta$.
- Dimensional moment derivatives of $x$-axis due to change in $p, q$ and $\xi$.
- Dimensional moment derivatives of $y$-axis due to change in $w, q$ and $\eta$.
- Dimensional moment derivatives of $z$-axis due to change in $p, q$ and $\xi$.
- Moment of inertia about $x, y$ and $z$ axis. 
$I_{x y}, I_{y z}, I_{x=} \quad$ - Product of inertia about $\mathrm{yx}, \mathrm{yz}$ and $\mathrm{xz}$ axis.

HUD - Head-Up Display.

FAA - Federal Aviation Administration.

ALT - Altitude.

IAS - Indicated Airspeed. 